\title{
Gravitational Optics: \\ Self-phase modulation and harmonic cascades
}

\author{
J.T.Mendonçâึ \\ GoLP, Instituto Superior Técnico, \\ 1049-001 Lisboa, Portugal \\ Vitor Cardosol] \\ CENTRA, Departamento de Física, Instituto Superior Técnico, \\ Av. Rovisco Pais 1, 1049-001 Lisboa, Portugal
}

(Dated: November 20, 2018)

\begin{abstract}
Nonlinear wave interaction of low amplitude gravitational waves in flat space-time is considered. Analogy with optics is established. It is shown that the flat metric space-time is equivalent to a centro-symmetric optical medium, with no second order susceptibility. The lowest order nonlinear effects are those due to the third order nonlinearity and include self-phase modulation and high harmonic generation. These processes lead to an efficient energy dilution of the gravitational wave energy over an increasingly large spectral range.
\end{abstract}

PACS numbers: 04.30.Nk, 42.65.-k, 95.30.Sf

${ }^{*}$ Electronic address: titomend@ist.utl.pt

$\dagger$ Electronic address: vcardoso@fisica.ist.utl.pt 


\section{INTRODUCTION}

Gravitational radiation is a direct result of the theory of gravitation [1, 2] and, even if not yet directly observed, its existence has been indirectly inferred from the change of orbital parameters in binary star systems [3]. Potential gravitational wave sources [4] can emit intense and short bursts, such as collapsing massive binary stars or supernova explosions, and continuous waves of much lower amplitudes, such as stable binary stars or non-axisymmetric spinning stars.

It is well known that the Einstein's equation governing the gravitational field contains strong nonlinearities. The nonlinear effects will, of course, be more relevant to the short bursts, containing only a few cycles of radiation, and associated with the more catastrophic events. It is also known that nonlinear gravitational waves of the soliton type can be excited [5, 6, 7, 8]. We have learned, however, from the more common situations of nonlinear optics [9], that the excitation of solitons depends very critically on the balance between the dispersive and the nonlinear effects. Even if the gravitational solitons do not seem to display such a balance and behave much more like superpositions of linear waves [10], they nevertheless correspond to particular solutions. And, with the exception of the soliton representation of cosmological solutions [11, 12, 13, which are pertinent to the large scale

structure of the universe, it is very unlikely that solitons will spontaneously be excited by local gravitational wave sources.

It is therefore quite plausible in physical terms to consider the problem of gravitational wave-packets of arbitrary shape. This will be considered here. In order to understand their typical behavior, and to describe the main physical pictures of their spectral evolution, we concentrate here on the simplest possible situation: that of propagation in a flat space-time. We are not interested in the wave generation phenomena, for which there exists a standard powerful formalism [14 valid even in strongly curved regions, nor in the propagation of gravitational waves in strongly curved regions. Here we want to focus on regions far from the sources, where the background is approximately flat. This allows us to simplify considerably the problem but still retaining some important physical features. We will try then to establish clear analogies with the well known concepts of nonlinear optics. In particular, the nonlinear gravitational susceptibilities will be established and the nonlinear wave mixing processes will be considered. Explicit analytical solutions for the processes of self-phase 
modulation and of high-order harmonic cascades will be derived.

\section{BASIC EQUATIONS}

Einstein's equation for the gravitational field in the absence of matter is given by:

$$
R_{i k}=\Gamma_{i k, l}^{l}-\Gamma_{i l, k}^{l}+\Gamma_{i k}^{l} \Gamma_{l m}^{m}-\Gamma_{i l}^{m} \Gamma_{k m}^{l}=0
$$

where the comma stands for ordinary derivative, and the Christofell symbols are determined by the derivatives of the metric tensor elements, according to:

$$
\Gamma_{i k}^{l}=\frac{1}{2} g^{l m}\left(\frac{\partial g_{m i}}{\partial x^{k}}+\frac{\partial g_{m k}}{\partial x^{i}}-\frac{\partial g_{i k}}{\partial x^{m}}\right) .
$$

We are considering a flat space-time, perturbed by a small amplitude gravitational wave. This means that we can write:

$$
g_{i j}=\eta_{i j}+h_{i j}
$$

where $\left|h_{i j}\right| \ll 1$, and:

$$
\eta_{00}=1 \quad, \quad \eta_{i i}=-1 \quad(i=1,2,3) \quad, \quad \eta_{i j}=0 \quad(i \neq j)
$$

In this case, we can write:

$$
R_{i k}=R_{i k}^{L}+R_{i k}^{N L}
$$

where the linear term is:

$$
R_{i k}^{L}=\frac{1}{2} \eta^{l m}\left(\frac{\partial^{2} h_{m i}}{\partial x^{l} \partial x^{k}}+\frac{\partial^{2} h_{m k}}{\partial x^{l} \partial x^{i}}-\frac{\partial^{2} h_{i k}}{\partial x^{l} \partial x^{m}}-\frac{\partial^{2} h_{m l}}{\partial x^{k} \partial x^{i}}\right) .
$$

The nonlinear term $R_{i k}^{L N}$ contains second, third and higher order nonlinearities. This means that, in principle, we can have three, four and higher wave mixing processes. Let us then write:

$$
R_{i k}^{L N}=R_{i k}^{(2)}+R_{i k}^{(3)}+\ldots
$$

where we have, for the lowest order term: 


$$
R_{i k}^{(2)}=\frac{h^{l m}}{\eta^{l m}} R_{i k}^{L}+\frac{\eta^{l p}}{h^{l p}} R_{i k}^{(3)}+\frac{1}{2}\left[\frac{\partial h^{l m}}{\partial x^{l}}\left(\frac{\partial h_{m i}}{\partial x^{k}}+\frac{\partial h_{m k}}{\partial x^{i}}-\frac{\partial h_{i k}}{\partial x^{m}}\right)-\frac{\partial h^{l m}}{\partial x^{k}} \frac{\partial h_{m l}}{\partial x^{i}}\right]
$$

The third order nonlinearities are contained in:

$$
\begin{aligned}
R_{i k}^{(3)}=-\frac{1}{4} \eta^{n m} h^{l p}\left[\left(\frac{\partial h_{m i}}{\partial x^{l}}+\frac{\partial h^{m l}}{\partial x^{i}}\right.\right. & \left.-\frac{\partial h_{i l}}{\partial x^{m}}\right)\left(\frac{\partial h_{p k}}{\partial x^{n}}+\frac{\partial h_{p n}}{\partial x^{k}}-\frac{\partial h_{k n}}{\partial x^{p}}\right) \\
& \left.-\left(\frac{\partial h_{p i}}{\partial x^{k}}+\frac{\partial h_{p k}}{\partial x^{i}}-\frac{\partial h_{i k}}{\partial x^{p}}\right) \frac{\partial h_{n p}}{\partial x^{l}}\right] .
\end{aligned}
$$

We could go on to higher orders but as we assume $\left|h_{i j}\right| \ll 1$, these terms become less important. Furthermore, stopping at third order suffices, as we will show, to uncover some truly non-linear and interesting aspects of gravitation.

It is well known [2] that the linear term (6) can simply be written as:

$$
R_{i k}^{L} \simeq-\frac{1}{2} \partial^{\mu} \partial_{\mu} h_{i k}
$$

where the d'Alembert operator is:

$$
\partial^{\mu} \partial_{\mu}=\frac{\partial^{2}}{\partial x^{j} \partial x_{j}}=\eta^{j n} \frac{\partial^{2}}{\partial x^{j} \partial x^{n}} .
$$

This means that we can write the nonlinear wave equation as:

$$
\partial^{\mu} \partial_{\mu} h_{i k}=2\left[R_{i k}^{(2)}+R_{i k}^{(3)}+R_{i k}^{(4)}\right] .
$$

\section{NONLINEAR WAVE COUPLING}

Equation (12) predicts the possibility of several types of nonlinear gravitational wave coupling. This can be studied by assuming that several waves of the type:

$$
h_{i j}(n)=\epsilon_{i j}(n) a(n) \exp \left[i q_{k}(n) x^{k}\right]
$$

coexist in the same region of flat space-time. Here $a(n)$ are slowly varying amplitudes, $\epsilon_{i j}(n)$ are unit polarization tensors such that $\epsilon_{i j}^{*} \epsilon_{i j}=1$, and $q_{k}(n)$ are the four-wavevectors associated with the interacting waves $n=1,2,3 \ldots$

The total wave field, for real waves, will be given by: 


$$
h_{i j}=\sum_{n} h_{i j}(n)+c . c .
$$

In order to determine the evolution of the amplitudes $a(n)$, we can construct envelope equation, by noting that:

$$
\partial^{\mu} \partial_{\mu} h_{i j}(n) \simeq\left(-q_{k} q^{k}+i q_{k} \frac{\partial}{\partial x_{k}}\right) h_{i j}(n) .
$$

Assuming that the waves still obey the linear dispersion relation, valid in the limit $R_{i j}^{N L} \rightarrow 0$ :

$$
q_{k}(n) q^{k}(n)=0
$$

we obtain, for three wave mixing:

$$
i q_{k}(1) \frac{\partial}{\partial x_{k}} a(1)=v(1) a(2) a(3) \exp \left(i \Delta q_{k} x^{k}\right),
$$

where:

$$
\Delta q_{k}=q_{k}(2)+q_{k}(3)-q_{k}(1)
$$

The nonlinear coupling coefficient $v(1)$ is determined by $R_{i j}^{(2)}$, equation(8). It can be verified that $v(1)=0$, which means that the three wave coupling is forbidden. We can use the hard way, by calculating explicitly $R_{i j}^{(2)}$, or the easy way, by noting that flat spacetime is a centro-symmetric medium and therefore, like in optics, it has no second order susceptibility. We then proceed to the next order of nonlinearity, and write the envelope equation for the four wave mixing process:

$$
i q_{k}(1) \frac{\partial}{\partial x_{k}} a(1)=w(1) a^{*}(2) a(3) a(4) \exp \left(i \Delta q_{k} x^{k}\right)
$$

where now:

$$
\Delta q_{k}=q_{k}(3)+q_{k}(4)-q_{k}(1)-q_{k}(2)
$$

and the coupling coefficient $w(1)$ is determined by $R_{i j}^{(3)}$. Similar equations given the other three wave amplitudes. In order to derive explicit results, we assume wave propagation along a given direction $x^{1}=x$. We have: $i q_{k} x^{k}=i q_{0} x^{0}+i q_{1} x^{1}$. We will use: $q_{1}=q, q_{0}=-\Omega / c$, and $x^{0}=c t$, which then leads to: 


$$
\exp \left(i q_{k} x^{k}\right)=\exp (i q x-i \Omega t)
$$

The linear dispersion relation (16) is:

$$
q_{k} x^{k}=\frac{\Omega^{2}}{c^{2}}-q^{2}=0
$$

This shows that flat space-time is non-dispersive. This means that, for $\Omega(2)+\Omega(1)=$ $\Omega(1)+\Omega(2)$, we can guarantee a perfect phase matching:

$$
\Delta q_{k}=0
$$

We will also have:

$$
i q_{k} \frac{\partial}{\partial x_{k}}=i q_{k} \eta^{k j} \frac{\partial}{\partial x^{j}}=i q\left(\frac{\partial}{\partial x}+\frac{1}{c} \frac{\partial}{\partial t}\right) .
$$

We can now introduce a variable $z=x-c t$, such that:

$$
i q(1) \frac{\partial}{\partial z} a(1)=w(1) a^{*}(2) a(3) a(4) .
$$

Let us use equation (9) to calculate. The result is:

$$
\begin{array}{r}
w(1)=\frac{1}{4} \epsilon^{i k *} \eta^{n m} \epsilon^{l p *}(2)\left\{\left[q_{l}(3) \epsilon_{m i}(3)+q_{i}(3) \epsilon_{m l}(3)-q_{m}(3) \epsilon_{i l}(3)\right] .\right. \\
{\left[q_{n}(4) \epsilon_{p k}(4)+q_{k}(4) \epsilon_{p n}(4)-q_{p}(4) \epsilon_{k n}(4)\right]-q_{l}(4) \epsilon_{n p}(4)} \\
\left.\left[q_{k}(3) \epsilon_{p i}(3)+q_{i}(3) \epsilon_{p k}(3)-q_{k}(3) \epsilon_{i k}(3)\right]\right\}+ \text { permutation }(2,3,4) .
\end{array}
$$

This is a very complicated expression, where the last term represents two terms formally identical to the first one, and obtained by permutation of the indices 2,3 and 4 . It is important to notice that, in order of magnitude, we have $w(1) \simeq \Omega^{2} / c^{2}$.

\section{SELF-PHASE MODULATION}

An important particular case is obtained when the modes $n=1,2,3,4$ coincide. The mode coupling equation ( 19 ) is then reduced to:

$$
i q \frac{\partial}{\partial z} a=w|a|^{2} a
$$


It is instructive to compare it with the envelope equation for an optical pulse (for instance a laser pulse), with central frequency $\omega_{0}$, propagating in a nonlinear medium with group velocity $v_{0}$. Its electric field amplitude is described by the equation [9]:

$$
\left(\frac{\partial}{\partial x}+\frac{1}{v_{0}} \frac{\partial}{\partial t}\right) E_{0}=i \omega_{0} \alpha\left|E_{0}\right|^{2} E_{0} .
$$

where $E_{0}$ is the electric field amplitude and $\alpha$ is the nonlinear coupling coefficient:

$$
\alpha=\frac{\omega_{0}}{k_{0} c^{2}} \chi^{(2)}
$$

Notice that equation can be written in a similar form:

$$
\left(\frac{\partial}{\partial x}+\frac{1}{c} \frac{\partial}{\partial t}\right) a=-i \frac{w}{q}|a|^{2} a .
$$

Comparing with equation (28) we conclude that the equivalent nonlinear susceptibility of the flat space-time, which would be the gravitational wave version of the electromagnetic susceptibility $\chi^{(2)}$ is equal one, whereas in optical materials the typical value is $\chi^{(2)} \sim 10^{-16}$. This means that he gravitational field is extremely nonlinear and that we expect to observe the same nonlinear effects as in optics at a much lower wave amplitudes. In particular, we can follow the usual optical approach and obtain a wavepacket solution of the form:

$$
a(x, t)=a(z) \exp [i \phi(z, t)]
$$

where the nonlinear phase is:

$$
\phi(z, t)=\phi_{0}-\frac{w}{q}|a(z)|^{2} t
$$

This means that a gravitational wavepacket, even of very small amplitude, will suffer a selfphase modulation such that its central frequency $\Omega$ will not remain fixed but will change along propagation, according to:

$$
\Omega=\Omega(0)-\frac{\partial \phi}{\partial t}=\Omega(0)-\frac{w}{q c} t \frac{\partial}{\partial z}|a(z)|^{2} .
$$

For instance, if we have a gaussian gravitational wave pulse of the form:

$$
|a(z)|^{2}=a_{0}^{2} \exp \left(-\frac{z^{2}}{z_{0}^{2}}\right)
$$


we will obtain a gravitational wave frequency shift $\Delta \Omega=\Omega-\Omega(0)$ :

$$
\Delta \Omega=\frac{w}{q c} \frac{2 z}{z_{0}^{2}}|a(z)|^{2} t
$$

It contrast with what usually occurs in nonlinear optics, this frequency shift will be positive at the pulse front $(z>0)$, and negative at the pulse rear $(z<0)$. We also see that, for very large propagation times $t$ this shift can be quite significant, $\Delta \Omega \gg \Omega(0)$, even for weak gravitational perturbations, $a \ll 1$, due to the already noticed strong nonlinearities. To be quantitative, let's see what conditions have to be fulfilled in order to have the same

effect as in nonlinear optics, i.e., to have the same frequency shift $\frac{\Delta \Omega}{\Omega}{ }_{\text {opt }}=\frac{\Delta \Omega}{\Omega}$ grav . In order to satisfy this condition we have to have

$$
|a(z)|^{2} \sim\left|E_{0}\right|^{2} \chi_{\text {opt }}^{(2)} \frac{d_{\text {opt }}}{d_{\text {grav }}},
$$

where $d_{\text {opt }}$ is a length travelled by light in usual experiments, and $d_{\text {grav }}$ is the length travelled by a gravitational wave until it reaches the gravitational wave detector. As a typical example, we have $d_{\text {opt }} \sim 10^{-3} \mathrm{~m},\left|E_{0}\right|^{2} \chi_{\text {opt }}^{(2)} \sim 10^{-2}$. For these values, we get

$$
|a(z)| \sim \frac{6 \times 10^{-13}}{\left[d_{\text {grav }}(K p c)\right]^{1 / 2}},
$$

where the distance is now measured in $K p c$. For a gravitational wave produced by any reasonable astrophysical event, our best expectations [14] give $a \sim 10^{-21}$, as the wave arrives on Earth. But, along its path from the radiation source to the Earth, its amplitude will remain significantly higher over large distances. It seems therefore very likely that such an effect will indeed take place.

\section{HARMONIC CASCADE}

Another possibility of nonlinear gravitational wave coupling is the generation of a large spectrum of harmonics of the initial frequency $\Omega(0)$. Here, in principle, the sum over $n$ will extend to infinity:

$$
h_{i j}=\sum_{n=-\infty}^{\infty} h_{i j}(n) .
$$

We will also have: 


$$
q_{k}(n)=n q_{k}
$$

Four wave mixing between these different harmonics will occur, where a perfect phasematching condition can be verified:

$$
\Delta q_{k}=\left[q_{k}(2)+q_{k}(n-1)\right]-\left[q_{k}(1)+q_{k}(n)\right]=0 .
$$

For the present case, an envelope equation for the amplitudes of the different harmonics can be derived, in the same way as equation (19). Assuming that the fundamental and the first harmonics are dominant, $\mid h_{i j}(n=1,2) \gg h_{i j}(n \neq 1,2)$, we can retain only two of the mixing terms and get, for propagation along one given direction $x$ :

$$
i q(n)\left(\frac{\partial}{\partial x}+\frac{1}{c} \frac{\partial}{\partial t}\right) a(n)=w(n)\left[a^{*}(1) a(2) a(n-1)+a(1) a^{*}(2) a(n+1)\right] .
$$

where $w(n)$ is determined by an expression similar to equation (26). Let us now define:

$$
a^{*}(1) a(2)=I e^{i \delta}
$$

where $I$ and $\delta$ are real. In order to get order of magnitude analytical solutions, let us assume that $w(n) \propto q(n)$, and let us also neglect the amplitude variations of the dominant modes, which means that the intensity parameter $I$ is approximately constant. We are then led to:

$$
\frac{\partial}{\partial z} a(n)=i w\left[e^{i \delta} a(n-1)+e^{-i \delta} a(n+1)\right]
$$

with:

$$
w=-\frac{w(n)}{q(n)} I
$$

It is now convenient to introduce new amplitude variables $b(n)$, such that:

$$
a(n)=(-1)^{n / 2} e^{i n \delta} b(n),
$$

and we get:

$$
\frac{\partial}{\partial z}=w[b(n-1)-b(n+1)] .
$$


Using $\tau=w z$, we can reduce this equation to the well known recurrence relation for Bessel functions:

$$
\frac{\partial}{\partial \tau} b(n)=b(n-1)-b(n+1) .
$$

An adequate solution will then be given in terms of Bessel functions of the first kind $J_{n}(\tau)$, such as:

$$
b(n, \tau)=A J_{n}(\tau)+A^{\prime} J_{n-1}(\tau),
$$

where the two constants $A$ an $A^{\prime}$ are determined by the initial conditions:

$$
A=a(n=1, \tau=0) \quad, \quad A^{\prime}=a(n=2, \tau=0),
$$

and all the other harmonic wave amplitudes as assumed to be initially equal to zero: $a(n \neq$ $1,2 ; \tau=0)$. Notice that this solution satisfies energy conservation, in the sense that:

$$
\sum_{n=-\infty}^{\infty}|b(n, \tau)|^{2}=\text { const }
$$

It is then an adequate, if if particular, solution to the problem of harmonic generation of low amplitude gravitational waves propagating in a flat space-time.

\section{CONCLUSIONS}

Nonlinear wave coupling of gravitational waves was considered in this work. The problem of low amplitude waves in flat space-time was examined, in order to extract the main physical consequences with the simplest possible formal complexity and to establish clear connections with the well known effects occurring in nonlinear optics.

In particular, the gravitational equivalent to the optical second and third order susceptibility was derived. It was shown that the second order gravitational susceptibility is equal to zero and, consequently, the process of three nonlinear wave coupling is forbidden. This is not surprising because the flat space-time background considered here can be seen as a centro-symmetric material medium. This property will eventually disappear in curved space-time. 
In contrast, the gravitational third order susceptibility was shown to be equal to one, thus meaning that the flat space-time is a strongly nonlinear medium. As in optics, four wave mixing processes can then be considered. But, unlike the case of optical phenomena, these wave mixing processes can become relevant even for very low wave amplitudes. The particularly important cases of generation of harmonic cascades and of self phase modulation were considered, and explicit analytical solutions were derived. They show that a significant spectral broadening and spectral energy dilution can then take place, specially in the vicinity of radiation sources where the wave amplitudes are non-negligible. Such energy dilution is not considered in the usual estimates for gravitational wave detection [4, 15] and could eventually lower the prospects for direct observation of these waves near the Earth.

Finally, it should be noticed that self phase modulation occurring in optics can be described as a particular example of photon acceleration [16, 17]. In analogy, the present result showing the existence of self phase modulation of gravitational wave pulses could also be interpreted as graviton acceleration. This aspect will be explored in a future work.

[1] S. Weinberg, Gravitation and Cosmology, Wiley, New York (1972).

[2] L.D. Landau and E.M. Lifshitz, The Classical Theory of Fields, Butterworth-Heinemann, Oxford (1975).

[3] J.H. Taylor, Rev. Mod. Phys., 66, 711 (1994).

[4] L.P. Grishchuk, V.M. Lipunov, K.A. Postnov, M.E. Prokhorov and B.S. Sathyaprakash, Usp. Phys. Nauk, 171, 3 (2001) [Phys. Usp., 44, 1 (2001)].

[5] V.A. Belinskii and V.E. Zakharov, Zh. Eksp. Teor. Fiz., 75, 1955 (1978) [Sov. Phys. JETP, 48, 985 (1978)].

[6] B.J. Carr and E. Verdaguer, Phys. Rev. D, 28, 2995 (1983).

[7] J. Ibanez and E. Verdaguer, Phys. Rev. D, 31, 251 (1985).

[8] A. Feinstein and Ch. Charach, Class. Quantum Grav., 3 L5 (1986).

[9] R.W. Boyd, Nonlinear Optics, Academic Press, New York (1992).

[10] P.T. Boyd, J.M. Centrella and S.A. Klasky, Phys. Rev. D, 43, 379 (1991).

[11] V.A. Belinskii and V.E. Zakharov, Zh. Eksp. Teor. Fiz., 77, 3 (1979) [Sov. Phys. JETP, 50, 1 (1979)]. 
[12] G.F.R. Ellis and M.A.H. MacCallum, Commun. Math. Phys., 12, 108 (1969).

[13] A. Feinstein, Phys. Rev. D, 35, 3263 (1987).

[14] R. A. Isaacson, Phys. Rev., 166, 1263 (1968); K. S. Thorne, Rev. Mod. Phys, 52, 299 (1980); B. F. Schutz and F. Ricci, in GravitationaL Waves, by I. Ciufolini et al (Editors), (Institute of Physics Publishing, Bristol, 2001).

[15] I.D. Novikov and V.P. Frolov, Usp. Fiz. Nauk, 171, 307 (2001) [Phys. Uspekhi, 44, 291 (2001)].

[16] L.O. Silva and J.T. Mendonça, Opt. Commun., 196, 285 (2001).

[17] J.T. Mendonça, Theory of Photon Acceleration, Institute of Physics, Bristol (2001). 